\title{
Jahresinhaltsverzeichnis 62. Jahrgang 2014
}

Editorial

Ines Engelmann / Katrin Etzrodt

Mediale Geschlechterkonstruktion online und mögliche Ursachen. Eine Inhaltsanalyse der journalistischen Online-Berichterstattung über Politikerinnen und Politiker .......

Sven Engesser / Frank Esser / Carsten Reinemann / Sebastian Scherr / Jörg Matthes / Anke Wonneberger

Negativität in der Politikberichterstattung. Deutschland, Österreich und die Schweiz im Vergleich

Volker Gehrau

Kultivierung von Berufsvorstellungen durch Fernsehen bei Jugendlichen

Christoph Kuhlmann / Christina Schumann / Jens Wolling

„Ich will davon nichts mehr sehen und hören!“ Exploration des Phänomens

Themenverdrossenheit

Michael Meyen

Medialisierung des deutschen Spitzenfußballs. Eine Fallstudie zur Anpassung von sozialen Funktionssystemen an die Handlungslogik der Massenmedien

Michael Meyen

Theorie der Medialisierung. Eine Erwiderung auf Anna M. Theis-Berglmair

Christoph Neuberger

Konflikt, Konkurrenz und Kooperation: Interaktionsmodi in einer Theorie der dynamischen Netzwerköffentlichkeit

Magdalena Obermaier / Mario Haim / Carsten Reinemann

Emotionen bewegen? Ein Experiment zur Wirkung von Medienbeiträgen mit Emotionalisierungspotenzial auf Emotionen, politische Partizipationsabsichten und weiterführende Informationssuche.

Corinna Oschatz / Marcus Maurer / Jörg Haßler

(R)Evolution der Politikberichterstattung im Medienwandel? Die Inhalte von nachrichtenjournalistischen Online- und Offline-Angeboten im Vergleich ...

Matthias Potthoff / Siegfried Weischenberg

Inspiration und Identität. Bibliometrische Befunde zur

kommunikationswissenschaftlichen Fachgeschichte

Doreen Reifegerste / Patrick Rössler

Soziale Appelle in der Gesundheitskommunikation. Motivkategorien als Grundlage für die theoretische Integration und die Systematisierung empirischer Befunde

$4 / 606$

Anna M. Theis-Berglmair

Medialisierung, Systeme und Organisationen. Ein Re-Arrangement von Theorie und eine Replik auf Michael Meyens „Medialisierung des deutschen Spitzenfußballs“ in $M \& K_{3} / 2014$

Friedemann Vogel

Die Zukunft im Visier. Die mediale Selbstinszenierung der Bundeswehr gegenüber Jugendlichen aus der Perspektive engagierter Diskurslinguistik ... 
Ines C. Vogel / Karin Stengel / Marion Rahnke

Qualität der Fernsehberichterstattung über internationale Krisen und Konflikte. Ein

Vergleich zwischen öffentlich-rechtlichen und privaten Nachrichtenanbietern

Stefanie Wahl / Christoph Klimmt / Alexandra Sowka

Außerschulische Medienkompetenzarbeit. Akteure, Prioritäten, erlebte

Herausforderungen

Manuel Wendelin / Ines Engelmann / Julia Neubarth

Nachrichtenfaktoren und Themen in Nutzerrankings. Ein Vergleich der

journalistischen Nachrichtenauswahl und der Selektionsentscheidungen des

Publikums im Internet.

Reihe „Klassiker der Kommunikations- und Medienwissenschaft heute"

Thomas Wiedemann

Pierre Bourdieu. Ein internationaler Klassiker der Sozialwissenschaft mit Nutzen für

die Kommunikationswissenschaft

Reihe „Methodeninnovationen in der Kommunikationswissenschaft"

Paula Stehr / Laura Leißner / Friederike Schönhardt / Patrick Rössler

Parasoziale Meinungsführerschaft als methodische Herausforderung. Entwicklung eines Fragebogeninstruments zur Messung des Einflusses von Medienpersonen auf die politische Meinungs- und Einstellungsbildung

Literatur

Besprechungen

Jörg-Uwe Nieland

Marcus Bölz (2014): Fußballjournalismus. Eine medienethnographische Analyse

redaktioneller Arbeitsprozesse. Wiesbaden: Springer VS

Christoph G. Grimmer (2014): Kooperation oder Kontrolle? Eine empirische

Untersuchung zum Spannungsverhältnis von Pressesprechern in der Fußball-

Bundesliga und Journalisten. Köln: Herbert von Halem

Maria Löblich

Lutz Hachmeister / Dieter Anschlag (Hrsg.) (2013): Rundfunkpolitik und Netzpolitik.

Strukturwandel der Medienpolitik in Deutschland. Köln: Herbert von Halem

Hans Peter Bull (2013): Netzpolitik. Freiheit und Rechtsschutz im Internet. Baden-

Baden: Nomos

Jacob Jünger

Ulrike Ackermann (Hrsg.) (2013): Im Sog des Internets. Öffentlichkeit und Privatheit im digitalen Zeitalter. Frankfurt am Main: Humanities Online

Beatrice Dernbach

Christopher William Anderson (2013): Rebuilding the News. Metropolitan Journalism in the Digital Age. Philadelphia: Temple University Press

Wolfgang Mühl-Benninghaus

Jörg Becker (Hrsg.) (2013): Die Digitalisierung von Medien und Kultur. Mit einem Vorwort von Lothar Bisky. Wiesbaden: Springer VS 
Dennis Reineck

Markus Beiler (2013): Nachrichtensuche im Internet. Inhaltsanalyse zurjournalistischen Qualität von Nachrichtensuchmaschinen. Konstanz : UVK

$1 / 102$

Nicola Döring

Mathias Blanz / Arnd Florack / Ursula Piontkowski (Hrsg.) (2014): Kommunikation. Eine interdisziplinäre Einführung. Stuttgart: Kohlhammer

$4 / 658$

Oliver Leistert

Benedetta Brevini / Arne Hintz / Patrick McCurdy (Eds.) (2013): Beyond WikiLeaks. Implications for the Future of Communications, Journalism and Society. Basingstoke/ New York: Palgrave MacMillan ....

Franziska Linke

Marion Brüggemann (2013): Digitale Medien im Schulalltag. Eine qualitativ rekonstruktive Studie zum Medienhandeln und berufsbezogenen Orientierungen von Lehrkräften. München: kopaed

Karl-Nikolaus Peifer

Inka Frederike Brunn (2013): Cache me if you can. Verfassungsrechtliche Aspekte der urheberrechtlichen Einordnung von Suchmaschinen. Baden-Baden: Nomos

Kerstin Thummes

Michael Bürker (2013): „Die unsichtbaren Dritten“. Ein neues Modell zur Evaluation und Steuerung von Public Relations im strategischen Kommunikationsmanagement.

Wiesbaden: Springer VS

Joan Kristin Bleicher

Hendrik Buhl (2013): Tatort: Gesellschaftspolitische Themen in der Krimireihe.

Konstanz: UVK

Melanie Radue

Ulla Carlsson (Ed.) (2013): Freedom of Expression Revisited. Citizenship and Journalism in the Digital Era. Göteborg: Nordicom

Ramón Reichert

Tanja Carstensen / Christina Schachtner / Heidi Schelhowe / Raphael Beer (Hrsg.) (2014): Digitale Subjekte. Praktiken der Subjektivierung im Medienumbruch der

Gegenwart. Bielefeld: transcript ....

Marie Cronqvist

Monika Djerf-Pierre / Mats Ekström (Eds.) (2013): Swedish Broadcasting.

Communicative Ethos, Genres and Institutional Change. Göteborg: Nordicom

Joan Kristin Bleicher

Mario Doulis / Peter Ott (Hrsg.) (2013): Remediate. An den Rändern von Film, Netz und Archiv. Paderborn: Wilhelm Fink ....

Castulus Kolo

Michael Eble (2013): Medienmarken im Social Web. Wettbewerbsstrategien und Leistungsindikatoren von Online-Medien aus medienökonomischer Perspektive.

Münster: Lit

Sebastian Armbrust

Susanne Eichner / Lothar Mikos / Rainer Winter (Hrsg.) (2013): Transnationale Serienkultur. Theorie, Ästhetik, Narration und Rezeption neuer Fernsehserien.

Wiesbaden: Springer VS 


\section{Christoph Neuberger}

Sven Engesser (2013): Die Qualität des Partizipativen Journalismus im Web. Bausteine für ein integratives theoretisches Konzept und eine explanative empirische Analyse. Wiesbaden: Springer VS

Juliane Finger

Linda Erker / Klaus Kienesberger / Erich Vogl / Fritz Hausjell (Hrsg.) (2013): GedächtnisVerlust? Geschichtsvermittlung und -didaktik in der Mediengesellschaft. Köln: Halem

Stephanie Geise

Sebastian Feuß (2013): Auf den ersten Blick. Wie Medieninhalte wahrgenommen und rezipiert werden. Wiesbaden: Springer VS

Josef Trappel

Terry Flew (2013): Global Creative Industries. Cambridge: Polity

Mark Dang-Anh

Claudia Fraas / Stefan Meier / Christian Pentzold (Hrsg.) (2013): Online-Diskurse.

Theorien und Methoden transmedialer Online-Diskursforschung. Köln: Herbert von

Halem

Denise Sommer

Thomas N. Friemel (2013): Sozialpsychologie der Mediennutzung. Motive,

Charakteristiken und Wirkungen interpersonaler Kommunikation über

massenmediale Inhalte. Konstanz: UVK ....

Florian Muhle

Christian Fuchs (2014): Social Media. A critical introduction. Los Angeles et al.: Sage

Publications

Leif Kramp

Michael Haller (2014): Brauchen wir Zeitungen? Zehn Gründe, warum die Zeitungen untergehen. Und zehn Vorschläge, wie dies verhindert werden kann. Köln: Halem . . .

Mark D. Cole

Stefan Heilmann (2013): Anonymität für User-Generated Content?

Verfassungsrechtliche und einfach-gesetzliche Analyse der Informationspflichten für journalistisch-redaktionelle Angebote und andere Telemedien in $\$ \S 5$ TMG, 55 RStV.

Baden-Baden: Nomos

Georg Ruhrmann

Iris Herrmann-Giovanelli (2013): Wissenschaftskommunikation aus der Sicht von Forschenden. Eine qualitative Befragung in den Natur- und Sozialwissenschaften.

Konstanz: UVK

Franzisca Weder

Olaf Hoffjann / Simone Huck-Sandhu (Hrsg.) (2013): UnVergessene Diskurse. 20 Jahre

PR- und Organisationskommunikationsforschung. Wiesbaden: Springer VS

Jana Schmitt

Diana Ingenhoff (Hrsg.) (2013): Internationale PR-Forschung. Konstanz: UVK .

Christian Hißnauer

Olaf Jacobs / Theresa Lorenz (2014): Wissenschaft fürs Fernsehen. Dramaturgie -

Gestaltung - Darstellungsformen. Wiesbaden: Springer VS .... 


\section{Matthias Potthoff}

Constanze Jecker (2014): Entmans Framing-Ansatz. Theoretische Grundlegung und empirische Umsetzung. Konstanz: UVK

Daniel Hajok

Thorsten Junge (2013): Jugendmedienschutz und Medienerziehung im digitalen

Zeitalter. Eine explorative Studie zur Rolle der Eltern. Wiesbaden: Springer VS

Achim Baum

Matthias Karmasin / Matthias Rath / Barbara Thomaß (2013): Normativität in der Kommunikationswissenschaft. Wiesbaden: Springer VS ....

Nea Matzen

Elisabeth Klaus / Ulla Wischermann (2013): Journalistinnen. Eine Geschichte in

Biographien und Texten 1848-1990. Berlin: Lit

Fabian Prochazka / Philip Sinner

Benjamin Krämer (2013): Mediensozialisation. Theorie und Empirie zum Erwerb medienbezogener Dispositionen. Wiesbaden: Springer VS

Elke Grittmann

Florian Kreutzer (2013): Ausgänge aus der „Frauen-Falle“? Die Un-Vereinbarkeit von Familie und Beruf im Bild-Text-Diskurs. Bielefeld: transcript . ...

Christian Nuernbergk

Uwe Krüger (2013): Meinungsmacht. Der Einfluss von Eliten auf Leitmedien und AlphaJournalisten - eine kritische Netzwerkanalyse. Köln: Halem

Barbara Thomaß

Matthias Künzler (2013): Mediensystem Schweiz. Konstanz: UVK

Hans Peter Bull

Martin Kutscha / Sarah Thomé (2013): Grundrechtsschutz im Internet? Baden-Baden:

Nomos

Johanna Möller

Dennis Lichtenstein (2014): Europäische Identitäten. Eine vergleichende Untersuchung der Medienöffentlichkeiten ost- und westeuropäischer EU-Länder. Konstanz,

München: UVK

Rüdiger Funiok

Michael Litschka (2013): Medienethik als Wirtschaftsethik medialer Kommunikation.

Zur ethischen Rekonstruktion der Medienökonomie. München: Kopaed

Elisabeth Klaus

Margreth Lünenborg / Tanja Maier (2013): Gender Media Studies. Eine Einführung.

Konstanz: UVK

Manuel Puppis

Angelika M. Mayer (2013): Qualität im Zeitalter von TV 3.0. Die Debatte zum öffentlichrechtlichen Fernsehen. Wiesbaden: Springer VS

Martin Löffelholz

Denis McQuail (2013): Journalism and Society. London: Sage

Jens Vogelgesang

Wiebke Möhring / Daniela Schlütz (Hrsg.) (2013): Handbuch standardisierte

Erhebungsverfahren in der Kommunikationswissenschaft. Wiesbaden: Springer VS . . 
Michael Brüggemann

Cornelia Mothes (2014): Objektivität als professionelles Abgrenzungskriterium im Journalismus. Eine dissonanztheoretische Studie zum Informationsverhalten von Journalisten und Nicht-Journalisten. Baden-Baden: Nomos ....

Paško Bilić

Jan Müller (2013): Mechanisms of Trust. News Media in Democratic and Authoritarian Regimes. Frankfurt a. M./New York: Campus

Christian Strippel

Dhiraj Murthy (2013): Twitter. Social Communication in the Twitter Age. Cambridge:

Polity Press

Armin Scholl

Teresa Naab / Daniela Schlütz / Wiebke Möhring / Jörg Matthes (Hrsg.) (2013):

Standardisierung und Flexibilisierung als Herausforderungen der kommunikationsund publizistikwissenschaftlichen Forschung. Köln: Halem

Friederike Siller

Brian O'Neill / Elisabeth Staksrud / Sharon McLaughlin (Eds.) (2013): Towards a Better Internet for Children? Policy Pillars, Players and Paradoxes. Göteborg: Nordicom .......

Klaus Neumann-Braun

Ingrid Paus-Hasebrink / Sascha Trültzsch / Alois Pluschkowitz /Christine W. Wijnen

(Hrsg.) (2013): Integrative AV- und Online-Kommunikationsforschung. Perspektiven Positionen - Projekte. Baden-Baden: Nomos

Sonja Kretzschmar

Horst Pöttker / Anke Vehmeier (Hrsg.) (2013): Das verkannte Ressort. Probleme und

Perspektiven des Lokaljournalismus. Wiesbaden: Springer VS

Michael Haller

Colin Porlezza (2014): Gefährdete journalistische Unabhängigkeit. Zum wachsenden Einfluss von Werbung auf redaktionelle Inhalte. Konstanz, München: UVK

Beatrice Dernbach

Senja Post (2013): Wahrheitskriterien von Journalisten und Wissenschaftlern. Baden-

Baden: Nomos...

Peter Maurer

Thorsten Quandt / Jürgen Wilke / Christine Heimprecht / Thilo von Pape (2014):

Fernsehwelten. Auslandsnachrichten im deutschen Fernsehen. Wiesbaden: Springer

VS

Thomas Hoeren

Christian Rauda (2013): Recht der Computerspiele. München: C.H. Beck

Martin Welker

Konrad Scherfer / Helmut Volpers (Hrsg.) (2013): Methoden der Webwissenschaft.

Teil 1. Unter Mitarbeit von Birgit Pieplow und Miriam Schmitz. Berlin: Lit

Olaf Hoffjann

Thomas Schierl / Jörg Tropp (Hrsg.) (2013): Wert und Werte der Marketing-

Kommunikation. Köln: Halem

Sascha Hölig

Anne Schulz / Patrick Rössler (2013): Schweigespirale Online. Die Theorie der

öffentlichen Meinung und das Internet. Baden-Baden: Nomos 
Sven Kommer

Anne Schulze (2013): Internetwerbung und Kinder. Eine Rezeptionsanalyse.

Heidelberg: Springer VS

$3 / 480$

Wolfgang Seufert

Insa Sjurts (Hrsg.) (2014): Zehn Jahre sind ein Jahr. Kernthemen der

medienwirtschaftlichen Forschung der letzten Dekade. Baden-Baden: Nomos ...

$4 / 679$

Daniela Schlütz

Hartmut Stöckl (Hrsg.) (2013): Werbung - Keine Kunst!? Phänomene und Prozesse der Ästhetisierung von Werbekommunikation. Heidelberg: Universitätsverlag Winter . . .

Paul Murschetz

Tanja Storsul / Arne H. Krumsvik (Eds.) (2013): Media Innovations. A Multidisciplinary Study of Change. Göteborg: Nordicom

Wolfgang H. Swoboda

Simon Sturm (2013). Digitales Storytelling. Eine Einführung in neue Formen des Qualitätsjournalismus. Wiesbaden: Springer VS ....

Anna M. Theis-Berglmair

Peter Szyszka (Hrsg.) (2012): Alles nur Theater? Authentizität und Inszenierung in der Organisationskommunikation Köln: Halem

$4 / 682$

Jörg Tropp

Kerstin Thummes (2013): Täuschung in der strategischen Kommunikation. Eine

kommunikationswissenschaftliche Analyse. Wiesbaden: Springer VS ....

Bernadette Kneidinger

Kai Erik Trost (2013): Soziale Onlinenetzwerke und die Mediatisierung der Freundschaft. Eine qualitative Studie zur Bedeutung von Facebook für das Freundschaftskonzept Jugendlicher. Baden-Baden: Nomos

Roland Gimmler

Ulrike Wagner / Christa Gebel / Claudia Lampert (Hrsg.) (2013): Zwischen Anspruch und Alltagsbewältigung: Medienerziehung in der Familie. Berlin: Vistas ....

Christian Strippel

Katrin Weller / Axel Bruns / Jean Burgess / Merja Mahrt / Cornelius Puschmann (Eds.)

(2014): Twitter and Society. New York: Peter Lang

Robert Seifert

Jeffrey Wimmer (2013): Massenphänomen Computerspiele. Soziale, kulturelle und wirtschaftliche Aspekte. Konstanz: UVK

Günther Rager

Thomas Zerback (2013): Publizistische Vielfalt. Demokratischer Nutzen und

Einflussfaktoren. Konstanz: UVK

Elizabeth Prommer

Zillich, Anna Freya (2013): Fernsehen als Event, Köln: Halem

Zeitschriftenlese $1 / 115,2 / 308,3 / 489,4 / 687$

Literaturverzeichnis $1 / 137,2 / 359,3 / 540,4 / 743$ 
English Abstracts $1 / 171,2 / 364,3 / 556,4 / 749$

\section{Kommunikationsmodi}

zwischen Massen- und interpersonaler Kommunikation

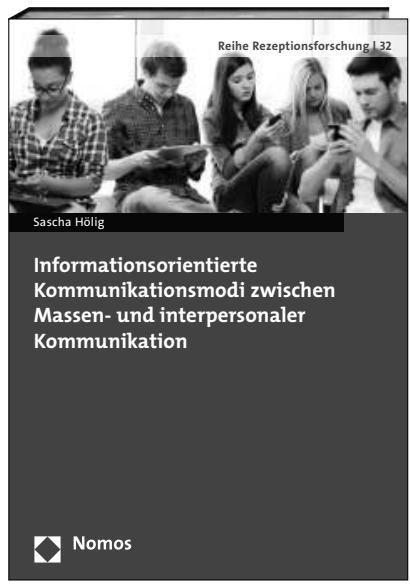

Informationsorientierte

Kommunikationsmodi

zwischen Massen- und inter-

personaler Kommunikation

Von Sascha Hölig

2014, 281 S., brosch., 49,- $€$

ISBN 978-3-8487-1814-6

(Reihe Rezeptionsforschung, Bd. 32)

www.nomos-shop.de/23558

Im Internet verschwimmen die Übergänge zwischen Massen- und interpersonaler Kommunikation. Dennoch unterscheiden Nutzer ihre Kommunikationshandlungen nach bestimmten Kriterien. Diese Kommunikationsmodi werden situationsspezifisch aktualisiert, je nachdem welche Funktion realisiert werden soll.

Bestellen Sie jetzt telefonisch unter 07221/2104-37

Portofreie Buch-Bestellungen unter www.nomos-shop.de

Alle Preise inkl. Mehrwertsteuer

Nomos 